\title{
Top Quark Properties Measurements with the ATLAS Detector
}

\author{
Reinhild Yvonne Peters, on behalf of the ATLAS Collaboration* \\ The University of Manchester \\ E-mail: repeters@cern.ch
}

\begin{abstract}
The top quark, discovered in 1995 by the CDF and D0 experiments at the Fermilab's Tevatron proton-antiproton collider, is the heaviest known elementary particle today. It has a life time shorter than the time for hadronisation, allowing its spin information to be accessed from its decay products. These features make the top quark a special particle to study. In this presentation, we focused on recent results from the ATLAS experiment in the top sector. In particular, we presented a new result on QCD colour-flow studies in top quark decays, a new measurement of spin correlations in top-antitop quark events, and new limits on the production of events with four top quarks. All results use a data sample of $36 \mathrm{fb}^{-1}$ of integrated luminosity, recorded with the ATLAS detector at $\sqrt{s}=13 \mathrm{TeV}$ collision energy.
\end{abstract}

ICHEP2018, 39th International Conference on High Energy Physics

4-11 July 2018

Seoul, Korea

${ }^{*}$ Speaker. 


\section{Introduction}

Discovered in 1995 by the Tevatron, the heaviest known elementary particle today is the top quark. Its mass is $m_{t}=172.51 \pm 0.5 \mathrm{GeV}$ according to the latest ATLAS combination [1]. The top quark has a life time that is shorter than the time scale for hadronization and the time scale for spin de-correlation effects. These features make the top quark an interesting object of study to scrutinise the Standard Model and search for new physics beyond the Standard Model.

In this proceedings, we outline three recent results on top quark physics. In particular, a new result on QCD colour flow in top antitop quark $(t \bar{t})$ events is presented, a new measurement of $t \bar{t}$ spin correlations, as well as new limits on the search for events containing four top quarks $(t \bar{t} t \bar{t})$. All analyses use $36 \mathrm{fb}^{-1}$ of ATLAS data, taken at $\sqrt{s}=13 \mathrm{TeV}$ collision energy.

\section{Measurement of QCD Colour Flow in $t \bar{t}$ Events}

One of the features of Quantum Chromo Dynamics (QCD) is that gluons carry two colour charges. Furthermore, only colour singlets are observed in nature. When two quarks are produced from the decay of a particle, as for example from a gluon or Higgs boson, these two quarks should be either connected to the initial state or to each other, depending on the colour-nature of the intermediate boson. The colour-connection influences the distribution of components within a jet. As discussed in Ref. [2], the colour structure of jets can be tested with a variable called "jet pull". The jet pull vector is a $p_{\mathrm{T}}$-weighted radial moment of the jet. By using pairs of jets, the jet pull angle can be defined by taking the angle between the pull vector and the connection vector between the centres of the two jets. In the latest ATLAS analysis four variables related to colour flow were measured in semileptonic $t \bar{t}$ events [3]. In particular, the relative pull angles of both jets from the $W$ boson decay were extracted, the jet pull magnitude of the higher-momentum jet of the $W$ boson decay, and the relative jet pull angle of the two $b$-tagged jets from top and antitop quark decays. The jets from $W$ boson should show colour connection, while the two $b$-jets should not be connected to each other.
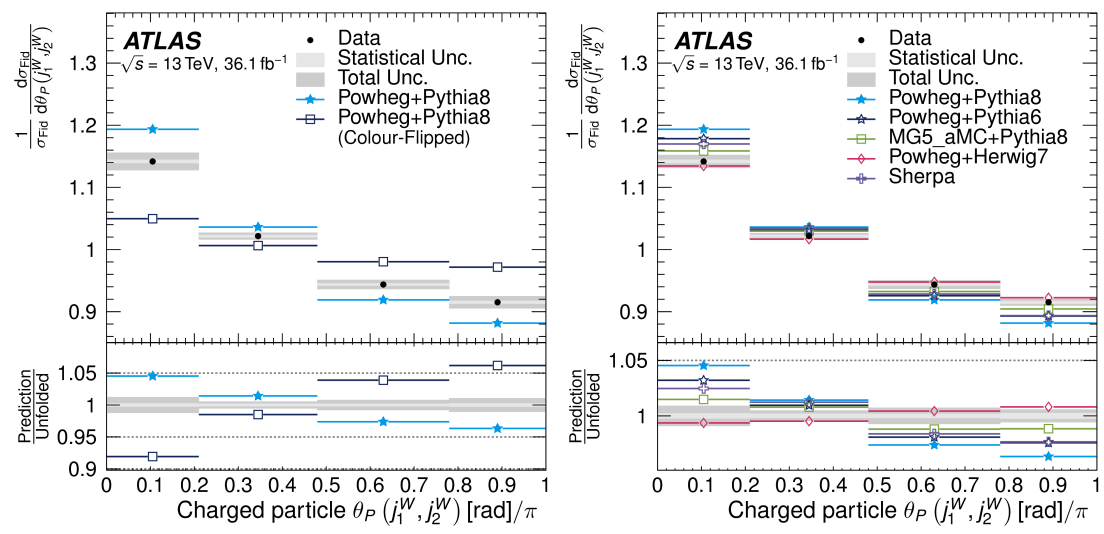

Figure 1: Example distributions of jet pull measurements for the higher- $p_{\mathrm{T}}$ jet [3]. Left: Relative jet pull angle compared to colour-flipped and standard $t \bar{t}$ MC. Right: Relative jet pull angle compared to several MC predictions. 

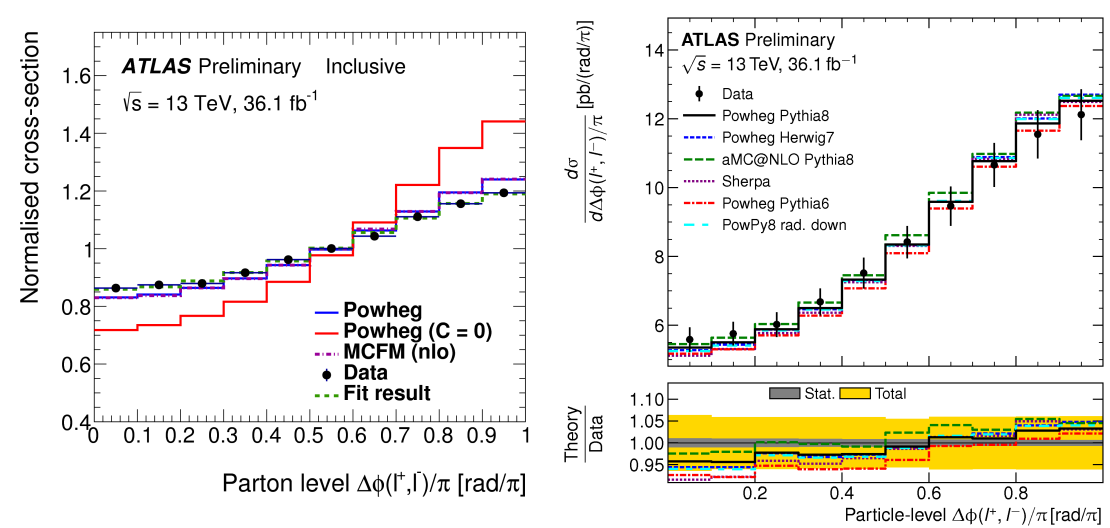

Figure 2: Distributions of $\Delta \phi$ at parton (left) and particle (right) level for the inclusive selections [4]. The parton-level distribution has MC predictions with and without $(C=0)$ spin correlations overlaid. The particle-level measurement shows comparisons with predictions from various MC generators.

The distributions of these observables were corrected back to stable particle level using iterative Bayesian unfolding, and are then compared to various Monte Carlo (MC) predictions, including a prediction with flipped colour-connections of the two jets from $W$ boson decay. The latter emulates the effect of a colour-octet-like $W$ boson decay, allowing to study the effect on the two jets without changing the kinematics of the event. Figure 1 shows example distributions of detector-corrected observables, with different MC predictions overlaid. In the measurement, it was found that the colour-flipped model is disfavoured in the data compared to the standard $t \bar{t}$ sample. Furthermore, it was found that none of the considered MC predictions can model all measured observables to a satisfying level.

\section{Measurement of $t \bar{t}$ Spin Correlations}

Due to the short lifetime of the top quark, information on its spin can be extracted from the decay products. In particular, the orientations of the spin of the top quark and antitop quark are correlated and can be studied by measuring angular observables in $t \bar{t}$ events. The measurement of the spin correlation is sensitive to new physics from production to decay.

The final state particle with the highest spin-analysing power is the charged lepton from $W$ boson decay. This motivates the use of dileptonic $t \bar{t}$ events for spin correlation studies. The main observable used to explore spin correlations at LHC is the difference in azimuthal angle between the two charged leptons from top decays, $\Delta \phi$, which has the advantage of being easy to measure and to not require information about the momenta of the two neutrinos.

In the recent analysis presented here, dileptonic events with one electron and one muon in the final state were used [4]. Differential distributions of $\Delta \phi$ were extracted in the inclusive sample and in bins of the invariant $t \bar{t}$ mass, $m_{t \bar{t}}$. The distributions were corrected for detector and acceptance effects, providing measurements at the parton and particle level. The unfolded distributions were then compared to various predictions from MC generators. Figure 2 shows the $\Delta \phi$ distribution for the inclusive measurement at parton and particle level, overlaid with predictions from different MC generators. The data show a shallower slope than any of the considered predictions. Comparing 

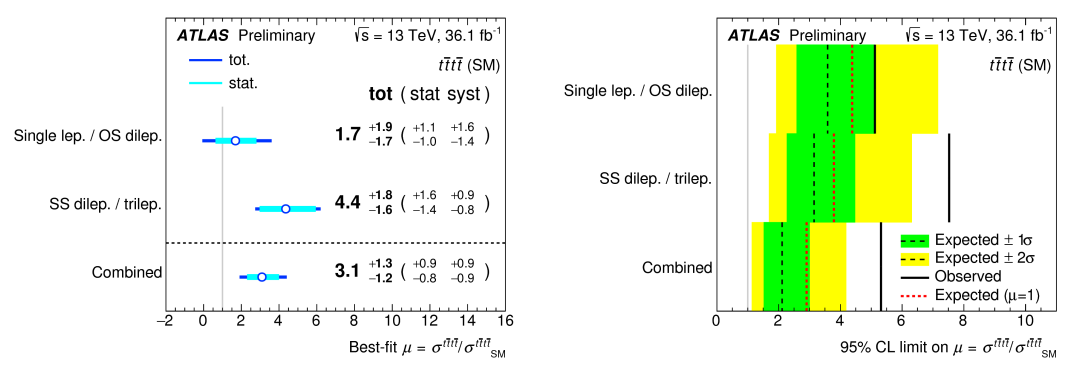

Figure 3: Measured value of $\mu$ (left) and limit on $\mu$ (right) for the production of $t \bar{t} t \bar{t}$ events [5, 6].

the MC predictions for a sample including Standard Model (SM) spin correlations with a sample assuming zero spin correlation, the extracted fraction of SM spin correlations is higher than one by 3.2 standard deviations.

\section{Searches for Events with Four Top Quarks}

With a SM cross section of $9.2 \mathrm{fb}$ at next-to-leading order, the production of $t \bar{t} t \bar{t}$ (four tops) events is very small. Several models of new physics can enhance the four top production, making it an interesting process to study. In a recent search for four top production, final states with one lepton and at least two leptons of same or opposite charge were considered [5, 6]. For each analysis, the events are categorised according to the number of leptons, $b$-jets, jets and the value of the missing transverse energy. Figure 3 shows the results of the likelihood fit for the individual final states and the combination, once shown as measurement of the ratio $\mu$ of measured cross section relative to the Standard Model cross section (left), and once interpreted as an upper limit on $\mu$ (right). The combined measurement of $\mu$ is above the SM expectation of $\mu=1$ with 2.8 standard deviations.

\section{Conclusion}

In this proceedings, three recent results in the top sector were presented. In particular, a sensitive analysis of QCD colour flow in $t \bar{t}$ events, a measurement of $t \bar{t}$ spin correlations, and new limits on the search for $t \bar{t} t \bar{t}$ production show interesting behaviors. With the increasing LHC data sample, and the ever-improving theoretical understanding, these analyses will become even more interesting in the future.

\section{References}

[1] ATLAS Collaboration, ATLAS-CONF-2017-071, Sept. 2017 (http://cdsweb.cern.ch/record/2285809).

[2] J. Gallicchio and M. D. Schwartz, Phys. Rev. Lett. 105 (2010) 022001.

[3] ATLAS Collaboration, arXiv:1805.02935, accepted by EPJC, May 2018

[4] ATLAS Collaboration, ATLAS-CONF-2018-027, July 2018 (https://cds.cern.ch/record/2628770).

[5] ATLAS collaboration, arXiv:1807.11883, submitted to JHEP, August 2018.

[6] ATLAS collaboration, arXiv:1811.02305, submitted to Phys. Rev. D, November 2018. 